\title{
Understanding Interfacial Water and Its Role on Various Practical Applications Using Molecular Simulations
}

\author{
Alberto Striolo \\ Department of Chemical Engineering \\ University College London \\ Torrington Place, London, WC1E 7JE, United Kingdom
}

\begin{abstract}
Interfacial water is believed to determine practical outcomes on systems of interest to biology, materials sciences, geology, and many other disciplines. Recent progress in understanding interfacial water achieved using molecular simulations is reviewed herein. After discussing the reliability of recent approaches, three possible research directions are discussed. These future developments promise large impact on both fundamental science and applications of societal importance.
\end{abstract}

\section{Introduction}

As demonstrated by this MRS Bulletin issue, understanding interfacial water remains a topic of great scientific and practical interest. The advancement in simulation algorithms and the availability of massive computational capabilities allow the community to improve such understanding. However, despite progress in algorithms, force fields, and computational capabilities, many open questions remain regarding interfacial water. Cross-comparison and validation between molecular simulation results and advanced experiments remains crucial, as is the enrichment of classic theoretical frameworks by the inclusion of new experimental and simulation results. This review is a succinct summary of recent simulation results regarding interfacial water, and a personal opinion regarding three directions along which future research could yield important societal benefits. Rather than discussing technical details (which can be found in authoritative references, some of which are provided), the article provides a general overview whose goal is to generate interest on the subject.

\section{Methods}

Simulation results could provide the bridge between theoretical and experimental approaches. The accuracy of molecular simulations relies on the availability of accurate force fields that describe the interactions among the various constituents of a system of interest. Much progress has been made in developing accurate force fields for water, ${ }^{1}$ although a single model able to describe all properties of bulk and interfacial water remains elusive. Many of the recent contributions on interfacial water have been obtained conducting molecular dynamics (MD) simulations. In MD the molecules are allowed to move within a carefully prepared simulation box, with their motion governed by Newton's laws of dynamics. ${ }^{2-4}$ 


\section{Background}

Attempts to relate the structure of interfacial water to macroscopic observables have a long and rich history. ${ }^{5}$ In 1959 Kauzmann used an analogy between protein folding in water and the transfer of a non-polar solvent from water to a reference non-polar solvent, ${ }^{6}$ and the wide implications of hydrophobic interactions were first discussed. Hydrophobic forces are due, at least in part, to the reorganization of water molecules within the hydration shell of a solute, which leads to a decrease in the system entropy. ${ }^{7-9}$ These phenomena are related to a variety of applications (protein folding and hydrocarbon solubility in water being just two well-known examples) in which water comes at contact with various solutes and surfaces. To benefit these applications, scientists have addressed questions such as: (1) how does the size of a hydrophobic solute contribute to the perturbation of hydration water? (2) How do ions perturb hydration water? (3) How do surface features (e.g., distribution of surface partial charges) affect hydration water? (4) How far from the solute does the perturbation persist? Etc.

Ashbaugh and Pratt made important contributions in quantifying the effect of solute size on the structure of interfacial water, and in using this perturbation to describe solute-solute interactions. ${ }^{10,11}$ They recently considered the effect of temperature. ${ }^{12}$ Because the reorganization of hydration water is more pronounced at low than at high $\mathrm{T}$, it would be expected that effective attractions between hydrophobic solutes (manifested experimentally via the second osmotic virial coefficient, $B_{22}$ ) is stronger at low T. Instead, their simulation results show that for molecular-size hydrophobic compounds $\mathrm{B}_{22}$ becomes more negative (more attractive) as T increases from 300 to $360 \mathrm{~K}$ ! This suggests that to positively affect practical applications it is not sufficient to analyze the molecular scale properties of interfacial water.

Simulations of simple ions hydration were conducted already two decades ago. Lee and Rasaiah considered a series of alkali metal ions and their halides in water at 298 $\mathrm{K}$, and showed that not only the structure of the hydration layer, but also the dynamics of water within the hydration shell influences the ions mobility in water. ${ }^{13}$ Smith and Dang considered the association of $\mathrm{NaCl}$ ion pairs in water, and highlighted the importance of water polarizability. ${ }^{14}$ More recently, the focus has moved towards multivalent ions. Dang and coworkers simulated $\mathrm{CaCl}_{2}, \mathrm{SrCl}_{2}, \mathrm{SrB}_{2}$, and $\operatorname{SrI}_{2} .^{15,16}$ Small changes in the parameterization of the ion-ion interaction potential strongly affect both ion association and ions accumulation at watervacuum interfaces. Unfortunately, limited experimental data are available to refine the interatomic potentials. Clearly, much needs to be done to achieve a molecularlevel interpretation of spectroscopic data suggesting that, e.g., ions are excluded from hydrophobic hydration shells, ${ }^{17}$ and that ion-specific effects manifest for amphiphiles hydration at interfaces. ${ }^{18}$ Regarding ion-specific effects, Lo Nostro and Ninham reviewed evidence such effects as observed in biology, ${ }^{19}$ and Jungwirth and Cremer reviewed the hydration structure of ions. ${ }^{20}$ While it is accepted that the structure of hydration water is related to macroscopic observables, its short-range nature suggests that other factors might also be important for obtaining a comprehensive understanding of these phenomena. 


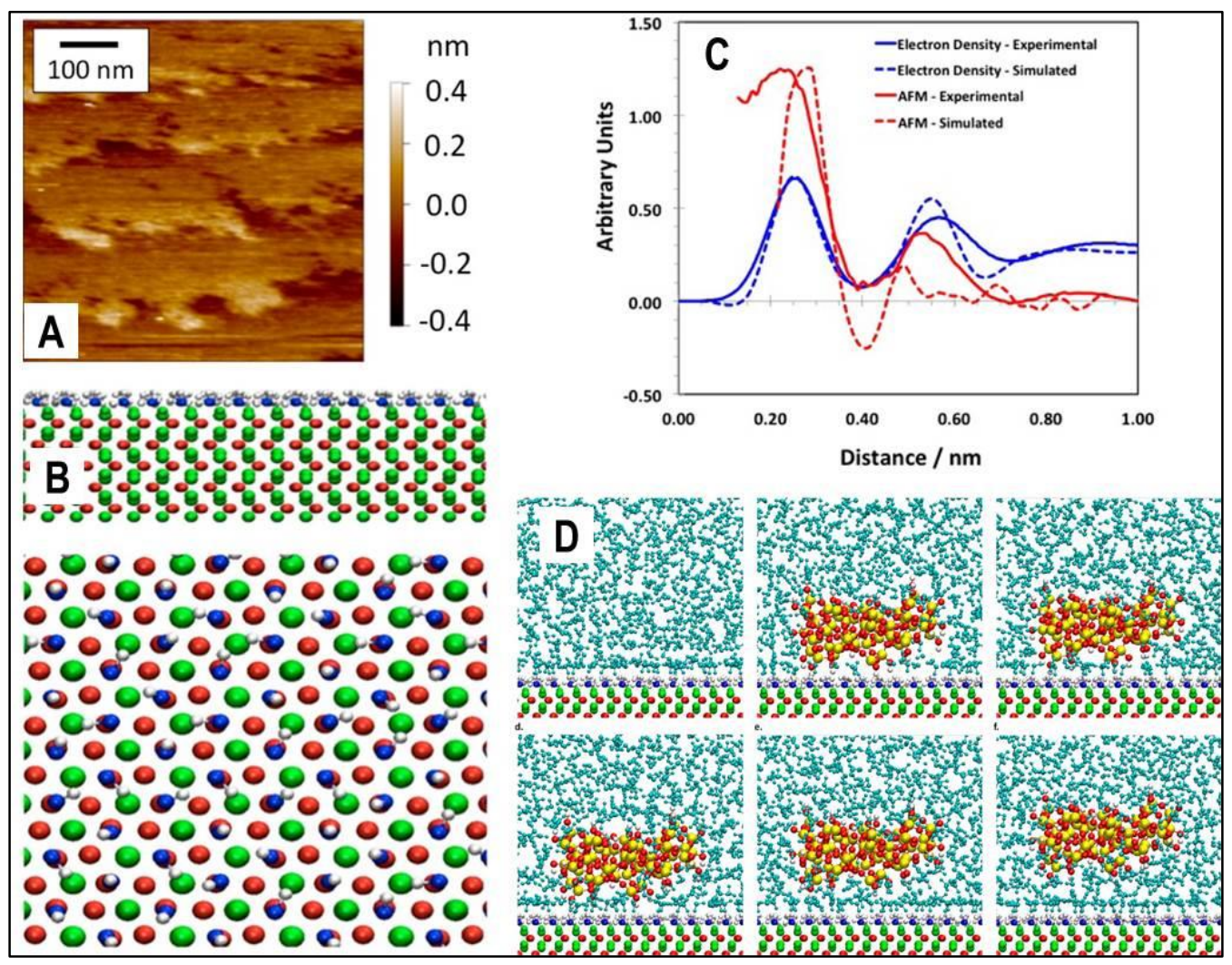

Figure 1. Panel A: AFM scan of the alumina crystal surface used for sampling the structure of interfacial water. Panel B: Simulation model for the alumina crystal.

Panel C: Comparison between experimental (continuous lines) and simulated (broken lines) data for the density of interfacial water (blue) and the force-distance curve (red). Panel D: Simulation model for a small silica disc (amorphous) at various distances form the alumina substrate, used to extract the simulated force-distance curve via the umbrella sampling algorithm. Reproduced with permission from J.

Phys. Chem. C 117 (2013) 10433.

To quantify how a solid substrate perturbs structure and dynamics of interfacial water, it has become routine to simulate a thin water film supported on a solid. ${ }^{21}$ The data generally show the formation of a dense first hydration layer when the solid substrate is atomically smooth; in some cases a second hydration layer is observed; bulk water density is recovered at distances larger than $\sim 1.2 \mathrm{~nm}$. It might be a surprise that similar features are observed also on graphitic substrates. ${ }^{22-24}$ When possible, water molecules interact with surface chemical groups, e.g. via hydrogen bonds. These water molecules can act as 'anchors' for molecules in the second hydration layer, which are less strongly adsorbed than those in the first layer. In Figure 1 we reproduce a comparison between simulations and experiments for water on crystalline alumina. ${ }^{25}$ Experiments are from X-ray reflectivity, ${ }^{26,27}$ or from an atomic force microscope. The density of the first hydration layer as predicted by $\mathrm{MD}^{28}$ is in quantitative agreement with experimental X-ray reflectivity. 
The force-distance profile obtained using umbrella sampling between a small amorphous $\mathrm{SiO}_{2}$ disc approaching the alumina substrate shows good qualitative agreement with experimental AFM data within the first repulsive peak. The comparison between simulated and experimental data in the second hydration layer suggests that improvements are needed. In general, simulations are conducted for a rigid substrate, while it is known that the atoms at the solid interface rearrange compared to the bulk crystalline structure. The solid-water interaction potentials need to be improved. It is likely that even the water-water force fields need to be improved to properly describe the interfacial region. Despite these limitations, we conclude that simulations are likely to reproduce experimental observables when accurate force fields are available (the state of the art regarding clay-water force fields is described elsewhere in this issue ${ }^{29}$ ). While qualitative features for interfacial water have been reported to be similar for interfacial water at contact with different substrates, details differ. In Figure 2 we reproduce rather old, but still insightful simulation data for water on talc (001), a prototypical 'hydrophobic' substrate, and muscovite (001), considered to be 'hydrophilic'. ${ }^{30}$

The perturbation of dynamical properties of interfacial water are consistent with structural perturbations, and strongly depend on the substrate; water shows faster tangential diffusion near surfaces that do not attract water, and vice versa, slower tangential diffusion near attractive surfaces. ${ }^{21}$ Regarding the relation between structure and dynamics, a recent investigation shows that water shear can lead to substantial changes in surface charges, and possibly to a reconstruction of the electric double layer, as a result of dissolution processes on surfaces of materials with low solubility in water. ${ }^{31,32}$

Returning to atomistic simulations for interfacial water, results showed that the distribution of preferential adsorption sites on the surface strongly affects the first hydration layer. In some cases, patterns of preferential adsorption sites can yield extended long-lasting hydrogen-bonded networks. These structures, which could yield strong repulsive forces against approaching molecules, ${ }^{33}$ could help preventing surface fouling. ${ }^{34}$ Studies have been reported concerning how the distribution of preferential interaction sites on a surface affects the water-solid interfacial tension, ${ }^{35}$ and how interfacial water structure determines hydrodynamic slip. ${ }^{36}$

To relate short-range differences such as those shown in Figure 2 to markedly different macroscopic properties, Garde, Chandler and coworkers proposed to quantify the water density fluctuations in the interfacial region. ${ }^{37-40}$ In short, it is more likely for water molecules to yield instantaneous empty pockets near 'hydrophobic' than near 'hydrophilic' substrates. Density fluctuations are correlated to the ease of molecules adsorption, ${ }^{41}$ and to the thermodynamics and kinetics of hydrophobic interactions at interfaces. ${ }^{42}$ This new tool is promising for enabling simulations to aid the development of new materials, e.g., 'translucent molecular coatings' based on graphene, ${ }^{43,44}$ and other practical applications. 


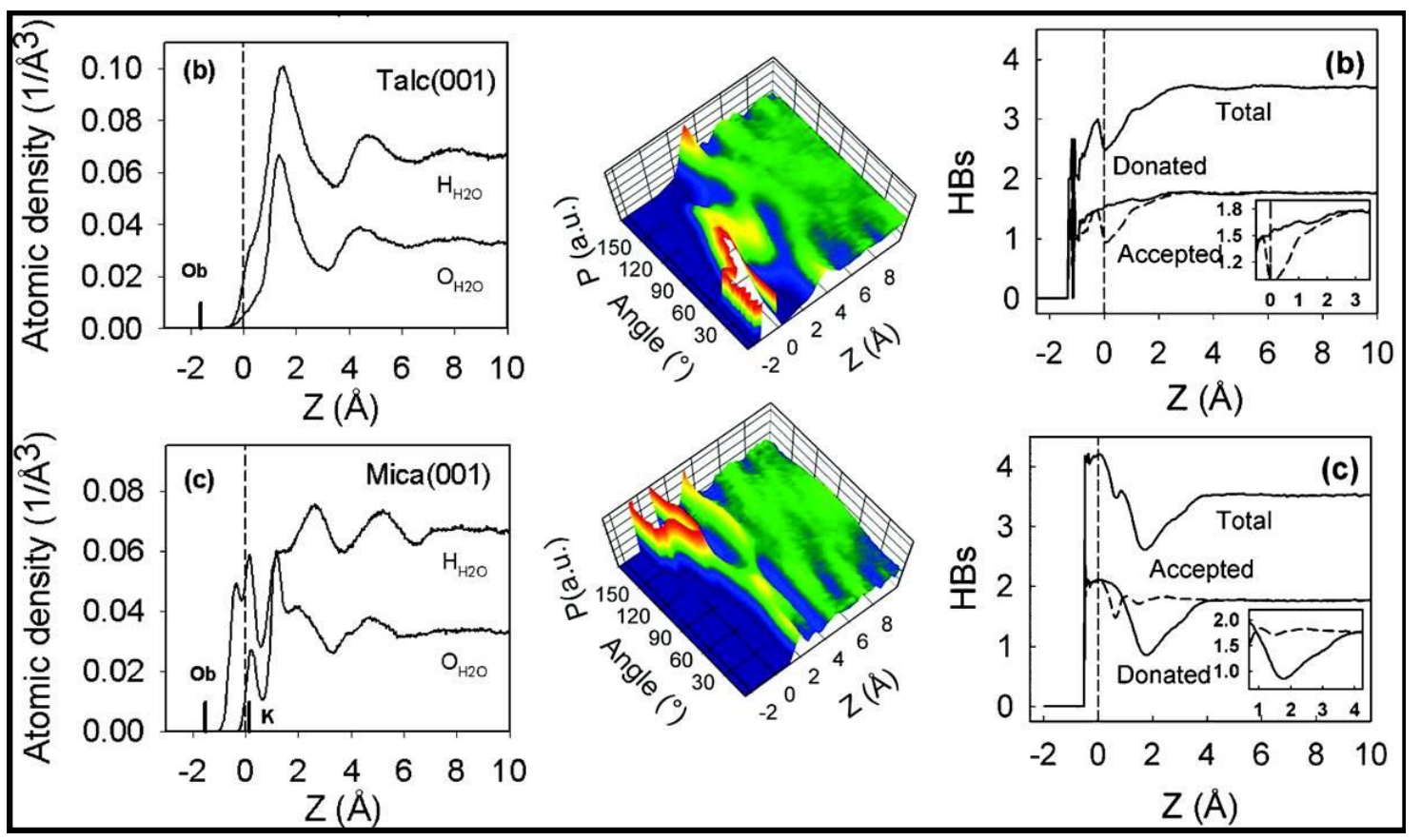

Figure 2. Top: data obtained for water at contact with talc (001). Bottom: data obtained on mica (001). From left to right the results represent atomic density profiles as a function of the distance from the surface (for both $\mathrm{O}$ and $\mathrm{H}$ atoms of water), probability distribution of the dipole moment of water molecules as a function of the distance from the substrate, and number of hydrogen bonds per water molecules (donated and received) as a function of the distance from the surface. Reproduced with permission from J. Phys. Chem. C 113 (2009) 11077.

\section{Possible Extensions to Practical Applications}

We just discussed recent progress on quantifying the properties of interfacial water and classifying systematically surfaces depending on their water affinity. In this section three possible extensions of fundamental research towards practical applications are highlighted. 


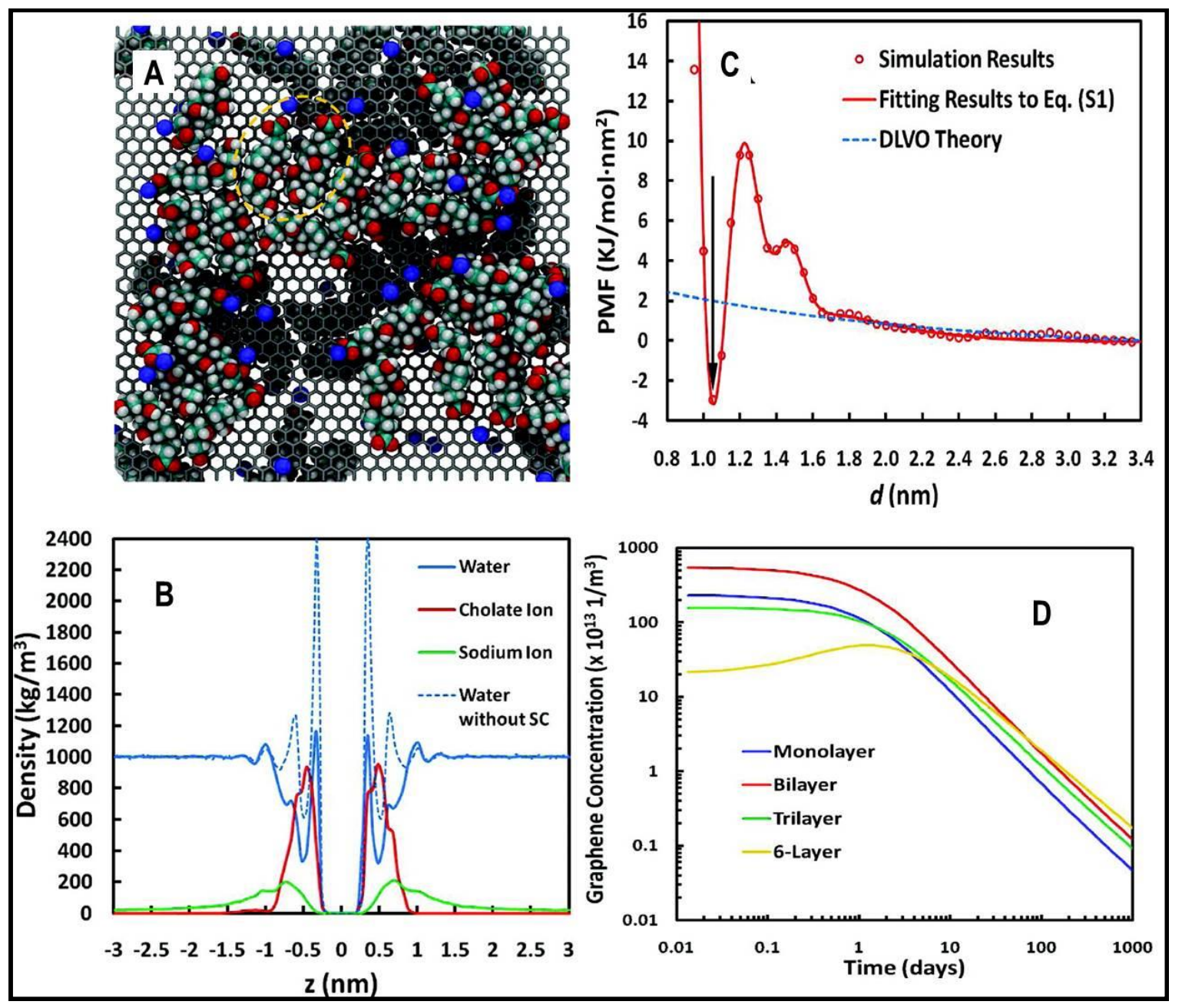

Figure 3. Panel A: simulation snapshot for sodium cholate surfactants adsorbed on one graphene sheet. Panel B: Density distribution in the direction perpendicular to the graphene sheet of water molecules, sodium cholate ions, and sodium ions. Panel

C: Simulation results for the graphene-graphene free energy profile (potential of mean force) as a function of the distance between the graphene layers, fitting of the simulation data to a mathematical function, and fitting to the DLVO theory. Panel D: prediction of the concentration as a function of time of single-, dual- tri-, and multilayer graphene sheets in an aqueous dispersion. Reproduced with permission from J. Am. Chem. Soc. 133 (2011) 12810.

A) Self-Assembly of Nanostructures

The structure of hydration water, and its relation to hydrophobic effects are considered instrumental for the self-assembly of surfactants, proteins, and nanostructures. To secure progress, a quantifiable relationship is required between interfacial water properties and effective interactions in water. De Benedetti and coworkers investigated the mechanism of graphene aggregation in water, and showed that such mechanism depends on the platelets size. ${ }^{45,46}$ When the platelets 
are of $3 \mathrm{~nm}$, an empty cylinder that spans the distance between the two platelets needs to form in water to promote aggregation. When the platelets are of $1 \mathrm{~nm}$, agglomeration occurs when all the water molecules in between spontaneously evaporate ${ }^{47}$ Accurate descriptions of the free energy landscapes correspondent to these mechanisms have been provided. We believe that practical applications will benefit enormously when we will learn to systematically translate such free energy profiles to macroscopic models for the kinetics of agglomeration. Blankschtein and Strano, e.g., combined free energy profiles from atomistic simulations with kinetic models of colloids agglomeration to describe the aggregation of graphene sheets stabilized by surfactants. ${ }^{48,49}$ In Figure 3 we reproduce their results. Comparison against experiments is acceptable, although the approach relies on one adjustable parameter. Future research could aim at determining such parameter by simulation insights. This would allow researchers to design methods for the stabilization of particles in solution, and perhaps control their assembly at desired conditions.

B) Membranes and Separation Processes

Membranes are used in many separation processes. Simulations could be used to better understand the mechanisms involved when various substances diffuse through membranes impregnated with water. ${ }^{50}$ Water-impregnated porous systems, such as membranes, are important for many current and future applications. For example:

(1) In hydrogen fuel cells protons diffuse through (poly) persulfonic acid membranes (e.g., Nafion $囚)$ soaked in water. Simulations are difficult because the structure of Nafion $囚$ is not clear at the molecular level. ${ }^{51}$

(2) Understanding how the diffusion of water is coupled to that of vitamins and perhaps drugs could be beneficial for designing soft contact lenses. While experiments are available, ${ }^{52}$ simulations for these systems are not common.

(3) To exploit shale gas in environmentally friendly ways it appears necessary to quantify the coupled transport of water, hydrocarbons, electrolytes, and environmental contaminants through the narrow pores present in shale formations, widely available worldwide. ${ }^{53}$

To bring such efforts to practical fruition it is necessary to understand how the thermodynamic properties of confined water ${ }^{54,55}$ are coupled with the transport of water and other compounds. It is also necessary to quantify how the thermodynamic properties of aqueous systems change upon confinement. The results summarized in Figure 4, e.g., suggest much larger methane solubility in water under confinement, and perhaps the presence of short-lived methane hydrates at conditions that prevent hydrates formation in bulk. ${ }^{56,57}$ Careful experimental characterization of the membranes (and/or of the confining matrices) is essential for these simulations to bear the promised fruits. 


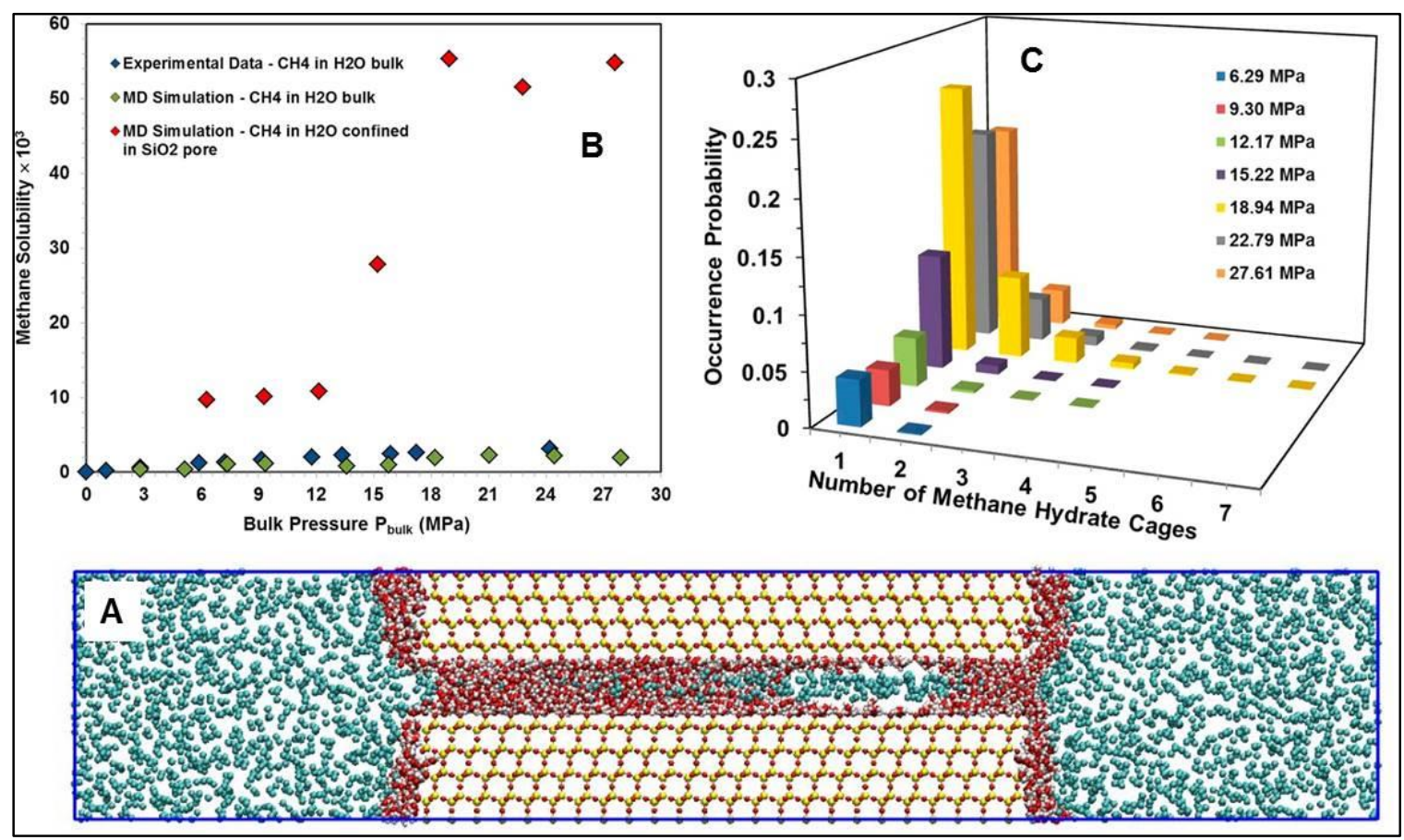

Figure 4. Panel A: System used to simulate the solubility of methane in water confined within a $1 \mathrm{~nm}$ - wide slit-shaped pore carved out of silica. Panel B:

Solubility of methane in confined water (red) compared to the solubility in bulk water as a function of pressure. Panel C: Probability of observing hydrate cages in the confined system as a function of pressure. Reproduced with permission from J. Phys. Chem. C 118 (2014) 4860.

\section{C) Water-Energy Nexus}

Securing abundant fresh water and energy in economical yet environmentally friendly manners is one of the current scientific grand challenges. ${ }^{58,59}$ Water desalination will play a growing role, and electric double layer capacitors (EDLCs) could become relevant for deploying renewable energy sources. The performance of these technologies depends on the structure and dynamics of hydrated electrolytes near carbon supports. Atomistic simulations clarified some principles for designing graphene membranes suitable for water desalination; ${ }^{60,61}$ Merlet et al. ${ }^{62}$ discovered that the unusually high capacitance observed in porous carbons with extremely narrow pores could be due to the distribution of the ions within edge, planar, hollow, and pocket sites; combining experiments and simulations, Kalluri et al.63,64 showed that it is necessary to apply an ion-dependent large voltage to force ions into narrow pores. To advance these contributions, the next challenge is to integrate the information obtained from equilibrium and non-equilibrium molecular simulations into continuum approaches that describe charge-discharge cycles in realistic models for the electrodes. Bazant and coworkers ${ }^{65}$ proposed to solve the modified Poisson-Nernst-Planck model, while accounting for ion-ion and ionelectrode correlations through appropriate parameters. ${ }^{66}$ Future improvements should account for pore-entrance effects, for the ions diffusion within various pores, 
and for the eventual presence of preferential adsorption sites. Because complex electrolytes are often used in EDLCs, and because multivalent ions can be found in salty water, it will be critical to include in these approaches details regarding the hydration of both ions and hydrophobic compounds summarized in the Background section above.

\section{Conclusions}

The enormous progress made in the development of computing power, algorithms, and force fields allows modern researchers to implement molecular simulations for investigating interfacial water. Although the results must be handled and interpreted with care, as pitfalls are often possible, the picture provided by simulation results seems in most cases to be consistent, sometimes quantitatively, with experimental observations. More research needs to be conducted before simulation results can be trusted completely, but the state of the art already suggests that, in addition to providing valuable tools for interpreting experimental observations, simulations could be used to explore new phenomena, and perhaps guide experiments towards the development of new materials and processes. In my opinion, efforts devoted to relate interfacial water properties to self-assembly of nano-structured materials, to design advanced separation strategies, and to contribute to the solution of the water-energy nexus could bear fruit in the not-toodistant future.

\section{Acknowledgments}

The author is grateful to several agencies for generous financial support. In particular, the US Department of Energy, the US National Science Foundation, the Sloane Foundation (via the Deep Carbon Observatory), and the EU Marie Curie Career Integration Grant are thankfully acknowledged.

\section{References}

1. C. Vega and J.L.F. Abascal, Phys. Chem. Chem. Phys. 13, 19663 (2011).

2. M.P. Allen and D.J. Tildesley, Computer Simulation of Liquids (Oxford University Press, 1989).

3. D. Frenkel and B. Smit, Understanding Molecular Simulation: From Algorithms to Applications (Academic Press, 2002).

4. C.G. Gray, K.E. Gubbins, C.G. Joslin, Theory of Molecular Fluids (Oxford University Press, 2011).

5. A. Ben-Naim, Y. Marcus, J. Chem. Phys. 81, 2016 (1984).

6. W. Kauzmann, Adv. Protein Chem. 14, 1 (1959).

7. J.N. Israelachvili, Intermolecular and Surface Forces, 3rd ed. (Academic Press, 2011).

8. R.L. Baldwin, FEBS Lett. 587, 1062 (2013).

9. J. Wu and J.M. Prausnitz, Proc. Natl. Acad. Sci. U.S.A. 105, 9512 (2008).

10. H.S. Ashbaugh and L.R. Pratt, Rev. Modern Physics 78, 159 (2006).

11. H.S. Ashbaugh and L.R. Pratt, J. Phys. Chem. B 111, 9330 (2007). 
12. M.I. Chaudhari, S.A. Holleran, H.S. Ashbaugh, L.R. Pratt, Proc. Natl. Acad. Sci. U.S.A. 110, 20557 (2013).

13. S.H. Lee and J.C. Rasajah, J. Phys. Chem. 100, 1420 (1996).

14. D.E. Smith and L.X. Dang, J. Chem. Phys. 100, 3757 (1994).

15. L.X. Dang, T.B. Truong, B. Ginovska-Pangovska, J. Chem. Phys. 136, 126101 (2012).

16. L.X. Dang, X. Sun, B. Ginovska-Pangovska, H.V.R. Annapureddy, T.B. Truong, Faraday Disc. 160, 151 (2013).

17. B.M. Rankin and D. Ben-Amotz, J. Am. Chem. Soc. 135, 8818 (2013).

18. R. Scheu, Y. Chen, H.B. de Aguiar, B.M. Rankin, D. Ben-Amotz, S. Roke, J. Am. Chem. Soc. 136, 2040 (2014).

19.P. Lo Nostro and B.W. Ninham, Chem. Rev. 112, 2286 (2012).

20. P. Jungwirth, P.S. Cremer, Beyon Hofmeister, Nature Chem. 6, 261 (2014).

21. A. Striolo, Adsorpt. Sci. Technol. 29, 211 (2011).

22. A. Striolo, A.A. Chialvo, P.T. Cummings, K.E. Gubbins, Langmuir 19, 8583 (2003).

23. D. Argyris, N.R. Tummala, A. Striolo, D.R. Cole, J. Phys. Chem. C 112, 13587 (2008).

24. M.C. Gordillo and J. Marti, J. Phys.: Condens Matter 22, 284111 (2010).

25. D. Argyris, A. Phan, P.D. Ashby, A. Striolo, J. Phys. Chem. C 117, 10433 (2013).

26. J.C. Catalano, Geochim. Cosmochim. Acta 75, 2062 (2011).

27. J.C. Catalano, J. Phys. Chem. C 114, 6624 (2010).

28. A. Phan, T.A. Ho, D.R. Cole, A. Striolo, J. Phys. Chem. C 116, 15962 (2012).

29. MRS Bulletin paper on clay-water interfaces

30.J. Wang, A.G. Kalinichev, R.J. Kirkatrick, J. Phys. Chem. C 113, 11077 (2009).

31. G.A. Waychunas, Science 344, 1094 (2014).

32. D. Lis, E.H.G. Backus, J. Hunger, S.H. Parekh, M. Bonn, Science 344, 1138 (2014).

33. D. Argyris, P.D. Ashby, A. Striolo, ACS Nano 5, 2215 (2011).

34. R.M. Elder and A. Jayaraman, Soft Matter 9, 11521 (2013).

35.J. Wang, D. Bratko, A. Luzar, Proc. Natl. Acad. Sci. U.S.A. 108, 6734 (2011).

36. T.A. Ho, D.V. Papavassiliou, L.L. Lee, A. Striolo, Proc. Natl. Acad. Sci. U.S.A. 108, 16170 (2011).

37. S.N. Jamadagni, R. Godawat, S. Garde, Annu. Rev. Chem. Biomol. Eng. 2, 147 (2011).

38.S. Garde and A.J. Patel, Proc. Natl. Acad. Sci. U.S.A. 108, 16491 (2011).

39. A.J. Patel, P. Varilly, S.N. Jamadagni, H. Acharya, S. Garde, D. Chandler, Proc. Natl. Acad. Sci. U.S.A. 108, 17678 (2011).

40. A.J. Patel, P. Varilly, D. Chandler, S. Garde, J. Stat. Phys. 145, 265 (2011).

41.S.N. Jamadagni, R. Godawat, S. Garde, Langmuir 25, 13092 (2009).

42.S. Vembanur, A.J. Patel, S. Sarupria, S. Garde, J. Phys. Chem. B 117, 10261 (2013).

43.J. Rafiee, X. Mi, H. Gullapalli, A.V. Thomas, F. Yavari, Y.F. Shi, P.M. Ajayan, N.A. Koratkar, Nature Mater. 11, 217 (2012).

44. C.-J. Shih, M.S. Strano, D. Blankschtein, Nature Mater. 12, 866 (2013).

45. S. Sharma and P.G. Debenedetti, Proc. Natl. Acad. Sci. U.S.A. 109, 4365 (2012).

46. A.L. Ferguson, N. Giovambattista, P.J. Rossky, A.Z. Panagiotopoulos, P.G. Debenedetti, J. Chem. Phys. 137, 144501 (2012).

47.S. Sharma and P.G. Debenedetti, J. Phys. Chem. B 116, 13282 (2012).

48. S. Lin, C.-J. Shih, M.S. Strano, D. Blankschtein, J. Am. Chem. Soc. 133, 12810 (2011).

49. C.-J. Shih, S. Lin, M.S. Strano, D. Blankschtein, J. Am. Chem. Soc. 132, 14638 (2010).

50. A. Phan, D.R. Cole, A. Striolo, Langmuir (2014) in Press.

51. K.B. Daly, J.B. Benziger, P.G. Debenedetti, A.Z. Panagiotopoulos, J. Phys. Chem. B 117, 12649 (2013).

52. F. Fornasiero, F. Krull, J.M. Prausnitz, C.J. Radke, Biomaterials 26, 5704 (2005).

53. A. Yethiraj, A. Striolo, J. Phys. Chem. Lett. 4, 687 (2013).

54. A. Striolo, K.E. Gubbins, T.D. Burchell, J.M. Simonson, D.R. Cole, M.S. Gruszkiewicz, A.A. Chialvo, P.T. Cummings, Langmuir 21, 9457 (2005).

55. N. Giovambattista, P.J. Rossky, P.G. Debenedetti, Annu. Rev. Phys. Chem. 63, 179 (2012).

56. A. Phan, D.R. Cole, A. Striolo, J. Phys. Chem. C 118, 4860 (2014).

57. S.N. Chakraborty and L.D. Gelb, J. Phys. Chem. B 116, 2183 (2012). 
58. M. A. Shannon, P.W. Bohn, M. Elimelech, J.G. Georgiadis, B.J. Marinas, A.M. Mayes, Nature 452, 301 (2008).

59. J. M. Tour, C. Kittrell, V. L. Colvin, Nature Mater. 9, 871 (2010).

60. D. Cohen-Tanugi, J.C. Grossman, Nano Lett. 12, 3602 (2012).

61. D. Konatham, J. Yu, T.A. Ho, A. Striolo, Langmuir 29, 11884 (2013).

62. C. Merlet, C. Pean, B. Rotenberg, P.A. Madden, B. Daffos, P.L. Taberna, P. Simon, M. Salanne, Nature Commun. 4, 2701 (2013).

63. R.K. Kalluri, M.M. Biener, M.E. Suss, M.D. Merrill, M. Stadermann, J.D. Santiago, T.F. Baumann, J. Biener, A. Striolo, Phys. Chem. Chem. Phys. 15, 2309 (2013).

64. T.A. Ho, R.K. Kalluri, M.M. Biener, J. Biener, A. Striolo, J. Phys. Chem. C 117, 13609 (2013).

65. M.Z. Bazant, B.D. Storey, A.A. Kornyshev, Phys. Rev. Lett. 107, 046102 (2011).

66. P.H.R. Alijo, F.W. Tavares, E.C. Biscaia, Jr., A.R. Secchi, Langmuir (2014) in press. 